\title{
Non-nutritive artificial sweeteners as an emerging contaminant in environment: a global review and risks perspectives
}

\begin{abstract}
Generally, non-nutritive artificial sweeteners are widely utilized as sugar substitute in various applications. With various applications, non-nutritive artificial sweeteners are now being recognized as emerging contaminants with high water persistence and are chemically stable in environment. Although non-nutritive artificial sweeteners were documented on their occurrence in environment, yet their potential impacts to environment and human health remain ambiguous. Therefore, this review was prepared to provide a more comprehensive insight of non-nutritive artificial sweeteners in environment matrixes by highlighting special concerns on human health and environmental risks. Precisely, this review monitors the exploration of nonnutritive artificial sweeteners occurrences as an emerging contaminants in environment worldwide and their associated risks to human as well as environment. At present, there are a total of 24 non-nutritive artificial sweeteners' studies with regards to their occurrence in the environment from 38 locations globally, spanning across Europe including United Kingdoms, Canada, United States and Asia. Overall, the quantitative findings suggested that the occurrence of non-nutritive artificial sweeteners is present in surface water, tap water, groundwater, seawater, lakes and atmosphere. Among these environmental matrixes, surface water was found as the most studied matrix involving non-nutritive artificial sweeteners. However, findings on non-nutritive artificial sweeteners impacts on human health and environment are limited to understanding its overall potential impacts and risks. Additionally, this review also serves as a framework for future monitoring plans and environmental legislative to better control these emerging contaminants in environment.
\end{abstract}

Keyword: Non-nutritive artificial sweeteners; Emerging contaminant; Environmental matrixes; Human; Environment; Risks 